\title{
Mullins Effect for a Cylinder Subjected to Combined Extension and Torsion
}

\author{
Domenico De Tommasi • Giuseppe Puglisi
}

Received: 26 July 2006 / Accepted: 16 September 2006 /

Published online: 2 November 2006

(C) Springer Science + Business Media B.V. 2006

\begin{abstract}
We study the Mullins effect for a circular cylinder of incompressible, isotropic material under loading cycles of combined extension and torsion. The analysis is based on the constitutive model recently proposed in De Tommasi et al. (J. Rheol. 50: 495-512, 2006). This model assumes that the mechanical response at each material point results as a homogenized effect of a mixture of different materials with variable activation and breaking thresholds. We show the feasibility of this approach to treat complex, inhomogeneous deformations. In particular, we obtain for the generic loading path the analytical expressions of the stress field, of the axial force, and of the twisting moment. The proposed model exhibits the Mullins stress softening effect in the case of simple extension, simple torsion, and combined extension and torsion. We analyze in detail the path dependent behavior and the preconditioning effects.
\end{abstract}

Key words Mullins effect • rubberlike materials • softening • damage • preconditioning

Mathematics Subject Classifications (2000) 74 C15 • 74D10 • 74R99

\section{Introduction}

Rubber-like materials subjected to cyclic loading exhibit a stress-softening behavior, which is usually known as the Mullins effect. This material behavior was first analyzed by Bouasse and Carrière [3] and then extensively studied by Mullins in a number of papers $[9,10]$. From the microscopic point of view, the stress-softening is due to an alteration mechanism of the macromolecular network which is subjected to a continuous scission and cross-linking process. This behavior is particularly relevant

D. De Tommasi $(\varangle) \cdot$ G. Puglisi

Dipartimento di Ingegneria Civile e Ambientale, Politecnico di Bari, Bari, Italy e-mail: d.detommasi@poliba.it 
when filler particles are dispersed in the rubber, because there is a significant interaction between the filler and the macromolecular network.

Due to its theoretical and technological interest, today there is a wide literature on the Mullins effect including a number of phenomenological models (see [2] and [4] for an extensive reference list). Many of the proposed models can be traced back to the original approach of Mullins and Tobin [11] which considers a two phase material with a hard phase that transforms into a soft phase when the strain increases. In this vein, Wineman and Rajagopal [17] supposed that the material is a mixture of two macromolecular networks with different reference configurations. Later, the authors extended their model in order to consider a continuous transformation of the original network into new networks with variable reference configurations [13]. Finally, in a number of papers $[12,15,16]$ the authors have systematically applied their model to the analysis of special equilibrium problems. In these papers, the authors have also shown the possibility of describing other phenomena associated to the described Mullins effect, such as hysteresis, permanent strain, and healing.

A computationally efficient three-dimensional model has been recently proposed by Beatty and Krishnaswamy [2]. In this work the stress-softening behavior is modelled by considering an inelastic material with a memory of the 'maximum' past strain. The efficiency of this model in describing rubber bodies undergoing special deformations have been shown by the authors in a number of successive papers [1,7]. More recently, within the theory of limiting chain extensibility, Horgan et al. [5] and Horgan and Saccomandi [6] have proposed a mesoscopic model for the Mullins effect that describes the macromolecular network alteration by assuming an evolution law for the chains extensibility.

The analysis in the present paper is based on the model proposed in [4], where the authors derived a micromechanics based model for the Mullins effect which considers a mixture of two types of materials. The first one is elastic and allows a non-zero strength for any deformation. The complementary fraction is breakable and describes the network alteration. This fraction is assigned through a distribution of materials with variable activation and breaking thresholds. In particular, the stress is zero until the activation threshold is reached and also when the limit threshold is overcome. In [4] the authors discussed the predictability of the model by showing the effective possibility of determining the distribution function of the breakable materials by means of suitable loading histories.

In this paper we consider the application of the described model to the case of a circular cylinder subjected to an inhomogeneous deformation of combined torsion and extension. In particular we assume that the material is incompressible and that both elastic and breakable fractions have an energy function of the Neo-Hookean type. In addition, for the breakable fraction we assume that the activation and breaking thresholds depend on the first principal strain invariant. We remark that the model is suitable for the description of the ideal Mullins effect, neglecting other phenomena such as healing, recrosslinking, rate-dependence, and permanent strain that will be the subject of future work.

In Section 2 we describe the constitutive assumptions at the basis of the model for the rubberlike material. Then we apply this model to the combined deformation of extension and torsion of a circular cylinder (Section 3). In particular, we obtain explicit analytic expressions of the stress components, of the normal force, and of the twisting moment as functions of the loading history. In Section 4 we analyze the Mullins effect for the cases of simple extension, pure torsion, and combined 
extension and torsion. In particular, for each of these cases we consider loading cycles with increasing amplitude and we show that our model exhibits an "idealized Mullins effect" associated with increasing portions of broken material. As we show, the model delivers a remarkable path-dependence due to the dependence on the loading history of the mechanical response of the breakable fraction (i.e., along different paths we have different reference configurations and different fractions of activated and broken material). Finally, we describe both the preconditioning effects of previous elongation cycles on the torsion behavior and of previous torsion cycles on the simple extension loading.

\section{Constitutive Assumptions}

In this section we summarize our constitutive assumptions for the rubberlike material. We refer the reader to [4] for a detailed description of the constitutive properties of the model. As usual, let $\mathbf{X}$ be the reference configuration of a material point which, as a consequence of a deformation $\mathbf{f}$, occupies the position $\mathbf{x}=\mathbf{f}(\mathbf{X})$. Moreover we indicate with $\mathbf{F}=\nabla \mathbf{f}$ the deformation gradient and with $\mathbf{B}=\mathbf{F F}^{\mathrm{T}}$ the left Cauchy-Green strain tensor.

We suppose that at each material point $\mathbf{X}$ the mechanical response results as a homogenized effect of different types of material. In particular we assume that a fraction $\alpha$ of the material is elastic. Moreover, for the purpose of simplicity, we consider an isotropic, incompressible, and hyperelastic Neo-Hookean material whose energy density function $W_{e}$ is defined as

$$
W_{e}=\frac{\mu_{e}}{2}(I-3)
$$

so that the Cauchy stress $\mathbf{T}_{e}$ is given by

$$
\mathbf{T}_{e}=-p \mathbf{I}+\mu_{e} \mathbf{B},
$$

where $I$ is the first invariant of $\mathbf{B}$ and $p$ is an arbitrary pressure associated with the incompressibility constraint.

To take care of the network scission effects induced by the deformation, we suppose that the complementary fraction $(1-\alpha)$ of the material is breakable. Specifically, we suppose that this type of material is activated when the first invariant $I$ of $\mathbf{B}$ reaches an activation threshold $I_{a}$

$$
I=I_{a},
$$

whereas the material breaks when

$$
I=I_{b}
$$

with $I_{b}$ representing a breaking threshold, verifying $I_{b}>I_{a} \geq 3$ (notice that, as a consequence of incompressibility, $I \geq 3$ ). More precisely, the breakable material is activated when $\mathbf{F}=\mathbf{F}_{a}$ such that the first invariant $I$ of $\mathbf{B}_{a}:=\mathbf{F}_{a} \mathbf{F}_{a}^{T}$ satisfies the condition $I=I_{a}$. Moreover, we suppose that the elastic energy of the activated material and its Cauchy stress depend on the relative deformation gradient $\widehat{\mathbf{F}}:=\mathbf{F F}_{a}^{-1}$, so that the energy is a function of the first invariant $\widehat{I}$ of the relative left Cauchy-Green strain $\widehat{\mathbf{B}}:=\widehat{\mathbf{F F}}^{\mathrm{T}}$. (Here and in what follows we indicate with $(\widehat{.})$ the strain measures 
evaluated with respect to the activation configuration $\mathbf{F}_{a}$.) Analogously, the material breaks when $\mathbf{F}=\mathbf{F}_{b}$ such that the first invariant $I$ of $\mathbf{B}_{b}:=\mathbf{F}_{b} \mathbf{F}_{b}^{T}$ satisfies $I=I_{b}$.

Summarizing the previous hypotheses, the energy density $W_{b}$ of the breakable material is assigned as

$$
W_{b}= \begin{cases}0 & \text { if } I<I_{a}, \\ \frac{\mu_{b}}{2}(\widehat{I}-3) & \text { if } I_{a} \leq I \leq I_{b}, \\ \frac{\mu_{b}}{2}\left(\widehat{I_{b}}-3\right) & \text { if } I>I_{b},\end{cases}
$$

where $\widehat{I}_{b}$ is the invariant of $\widehat{\mathbf{B}}_{b}:=\widehat{\mathbf{F}}_{b} \widehat{\mathbf{F}}_{b}^{T}\left(\widehat{\mathbf{F}}_{b}:=\mathbf{F}_{b} \mathbf{F}_{a}^{-1}\right)$. As a consequence of Eq. 2.2, the Cauchy stress is given by

$$
\mathbf{T}_{b}= \begin{cases}\mathbf{0} & \text { if } I<I_{a}, \\ \mu_{b} \widehat{\mathbf{B}} & \text { if } I_{a} \leq I \leq I_{b}, \\ \mathbf{0} & \text { if } I>I_{b}\end{cases}
$$

Once the breakable material reaches the breaking threshold, we assume that it is unloaded for any successive loading. Observe that the damage is isotropic and it depends only on the maximum value attained by $I$ in the loading history.

As a main feature of our model, to take care phenomenologically of the properties of the polymeric network, made up of chains with different reference and limit "length", we suppose that the breakable fraction is composed of a distribution of materials with different activation and breaking thresholds. For the purpose of simplicity, in this paper we assume that for each breakable material the elastic domain is fixed, i.e.,

$$
I_{b}=I_{a}+\Delta,
$$

with $\Delta \geq 0$ a given material parameter (see [4] for a more general case). Under previous hypothesis, the breakable fraction of the material is defined by a scalar probability function $f=f\left(I_{a}\right)$ with

$$
f\left(I_{a}\right)=0 \text { for } I_{a}<3, \quad f\left(I_{a}\right) \geq 0 \text { for } I_{a} \geq 3, \quad \int_{3}^{\infty} f\left(I_{a}\right) d I_{a}=1 .
$$

Consider now a loading history $\mathbf{F}=\mathbf{F}(t), t \in[0, T]$ at a material point, and the associated histories $\mathbf{B}=\mathbf{B}(t)$ and $I=I(t)$. We first analyze the behavior of the material point, starting from the virgin configuration (characterized by no previous breaking event) when it is subjected to a strain path with $I(t)$ monotonically increasing. In this case $I(t)=I_{M}(t):=\max _{\tau \in(0, t)} I(\tau)$ for all $t$. In the following, we refer to this type of path as a primary loading path.

Along a primary loading path (as the path O-A in Figure 1) we may evaluate for the fraction $(1-\alpha)$ of breakable material the percentage $a$ of activated material and the percentage $b$ of broken material (see Figure 1b):

$$
a(I)=\int_{I-\Delta}^{I} f\left(I_{a}\right) d I_{a}, \quad b(I)=\int_{3}^{I-\Delta} f\left(I_{a}\right) d I_{a} .
$$




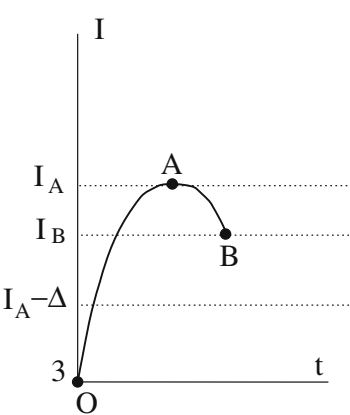

(a)

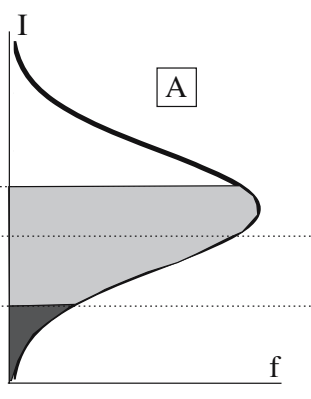

(b)

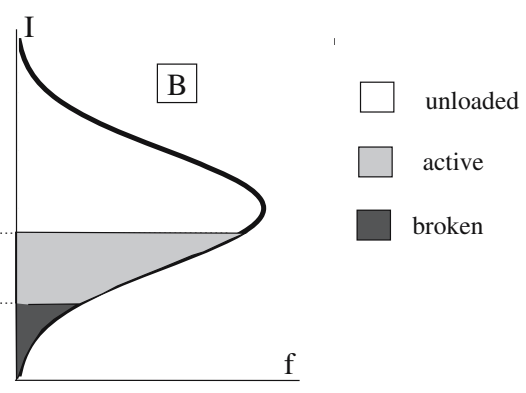

(c)

Figure 1 Evolution of the distribution of broken, active, and unloaded chains in the probability space $I \in(3, \infty)$ for the loading path $I=I(t)$ represented in (a). In (b) we show the distribution corresponding to the primary loading path $\left(I=I_{M}\right)$, in (c) the distribution after unloading $\left(I<I_{M}\right)$. Notice that for the loading paths with $I<I_{M}$ the fraction of broken chains is fixed and depends on the maximum previous value $I_{M}$, so that we obtain an elastic behavior (ideal Mullins effect).

Thus, according to Eqs. 2.1 and 2.3, based on an additivity hypothesis for the stress (see [4] for details), the Cauchy stress $\mathbf{T}$ may be evaluated as

$$
\mathbf{T}=-p \mathbf{I}+\alpha \mu_{e} \mathbf{B}+(1-\alpha) \mu_{b} \int_{I-\Delta}^{I} f\left(I_{a}\right) \widehat{\mathbf{B}} d I_{a}
$$

If we consider an unloading path, for which $I(t)<I_{M}(t)$, the activated fraction $a$ and the broken fraction $b$ can be evaluated as follows (see Figure 1c)

$$
a\left(I, I_{M}\right)=\int_{I_{M}-\Delta}^{I} f\left(I_{a}\right) d I_{a}, \quad b\left(I_{M}\right)=\int_{3}^{I_{M}-\Delta} f\left(I_{a}\right) d I_{a},
$$

Observe that along an unloading path no new chain is broken, and thus the behavior is elastic. In this case the Cauchy stress is given by

$$
\mathbf{T}=-p \mathbf{I}+\alpha \mu_{e} \mathbf{B}+(1-\alpha) \mu_{b} \int_{I_{M}-\Delta}^{I} f\left(I_{a}\right) \widehat{\mathbf{B}} d I_{a},
$$

for $I \geq I_{M}-\Delta$. For $I<I_{M}-\Delta$ only the elastic fraction $\alpha$ is active.

Note also that

$$
\text { if } I_{M}<3+\Delta \text {, then } b=0 \text {; }
$$

therefore $\Delta$ takes the role of an (overall) elastic threshold.

We remark that at the 'unloaded state' $(I=3)$ the memory of the system is restricted to the maximum past value $I_{M}$ of $I$. Moreover, as a consequence of our assumption of additivity of the stress, increasing the value of $I_{M}$ leads necessarily to a Mullins type softening effect because it corresponds to a growing percentage $b$ of broken material. 


\section{Extension and Torsion of a Circular Cylinder}

In this section we study the Mullins effect for a circular cylinder $\mathcal{B}$ constituted by a material of the type introduced in Section 2. The cylinder has reference length $L$ and outer radius $R_{o}$, and it is subjected to a combined effect of torsion and extension. We suppose that the lateral surface is unloaded.

We consider a cylindrical coordinate system and we denote with $(R, \Theta, Z)$ the coordinates of a material point $\mathbf{X}$ in the reference cylindrical configuration and with $(r, \theta, z)$ the coordinates of the corresponding point $\mathbf{x}$ in the current configuration. $\left(\mathbf{e}_{R}, \mathbf{e}_{\Theta}, \mathbf{e}_{3}\right)$ and $\left(\mathbf{e}_{r}, \mathbf{e}_{\theta}, \mathbf{e}_{3}\right)$ denote the reference and current cylindrical unit vector bases, respectively.

Consider the isochoric deformation

$$
r=\frac{R}{\sqrt{\lambda}}, \quad \theta=\Theta+\gamma Z, \quad z=\lambda Z
$$

with $\lambda \geq 1$ and $\gamma>0$, and $Z \in(0, L), \Theta \in(0,2 \pi), R \in\left(0, R_{o}\right)$. The deformation gradient $\mathbf{F}$ takes the form

$$
\mathbf{F}=\frac{1}{\sqrt{\lambda}} \mathbf{e}_{r} \otimes \mathbf{e}_{R}+\frac{1}{\sqrt{\lambda}} \mathbf{e}_{\theta} \otimes \mathbf{e}_{\Theta}+\lambda \mathbf{e}_{3} \otimes \mathbf{e}_{3}+r \gamma \mathbf{e}_{\theta} \otimes \mathbf{e}_{3},
$$

so that with respect to the current basis we have

$$
\mathbf{B}=\left[\begin{array}{ccc}
\frac{1}{\lambda} & 0 & 0 \\
0 & \frac{1}{\lambda}+r^{2} \gamma^{2} & r \gamma \lambda \\
0 & r \gamma \lambda & \lambda^{2}
\end{array}\right]
$$

this yields

$$
I=\frac{2}{\lambda}+\lambda^{2}+r^{2} \gamma^{2}
$$

To describe the behavior of the breakable material characterized by the activation threshold $I_{a}$, we need to evaluate the relative deformation gradient with respect to the activation deformation

$$
\mathbf{F}_{a}=\frac{1}{\sqrt{\lambda_{a}}} \mathbf{e}_{r_{a}} \otimes \mathbf{e}_{R}+\frac{1}{\sqrt{\lambda_{a}}} \mathbf{e}_{\theta_{a}} \otimes \mathbf{e}_{\Theta}+\lambda_{a} \mathbf{e}_{3} \otimes \mathbf{e}_{3}+\frac{R}{\sqrt{\lambda_{a}}} \gamma_{a} \mathbf{e}_{\theta_{a}} \otimes \mathbf{e}_{3},
$$

with $I\left(\lambda_{a}, \gamma_{a}\right)=I_{a}$ and $\left(\mathbf{e}_{r}^{a}, \mathbf{e}_{\theta}^{a}, \mathbf{e}_{3}\right)$ the current reference frame at $\mathbf{F}=\mathbf{F}_{a}$. Thus we get

$$
\mathbf{F}_{a}^{-1}=\sqrt{\lambda_{a}} \mathbf{e}_{R} \otimes \mathbf{e}_{r_{a}}+\sqrt{\lambda_{a}} \mathbf{e}_{\Theta} \otimes \mathbf{e}_{\theta_{a}}+\frac{1}{\lambda_{a}} \mathbf{e}_{3} \otimes \mathbf{e}_{3}-\frac{R}{\lambda_{a}} \gamma_{a} \mathbf{e}_{\Theta} \otimes \mathbf{e}_{3},
$$


so that the relative deformation gradient is given by

$$
\widehat{\mathbf{F}}:=\mathbf{F} \mathbf{F}_{a}^{-1}=\frac{1}{\sqrt{\hat{\lambda}}} \mathbf{e}_{r} \otimes \mathbf{e}_{r_{a}}+\frac{1}{\sqrt{\hat{\lambda}}} \mathbf{e}_{\theta} \otimes \mathbf{e}_{\theta_{a}}+\hat{\lambda} \mathbf{e}_{3} \otimes \mathbf{e}_{3}+r \hat{\gamma} \mathbf{e}_{\theta} \otimes \mathbf{e}_{3},
$$

where we have used the following measure of the relative strain

$$
\hat{\lambda}:=\frac{\lambda}{\lambda_{a}}, \quad \hat{\gamma}:=\frac{\gamma-\gamma_{a}}{\lambda_{a}}
$$

satisfying $\hat{\lambda}=1$ and $\hat{\gamma}=0$, when $\mathbf{F}=\mathbf{F}_{a}$. Finally, the relative left Cauchy-Green strain $\widehat{\mathbf{B}}:=\widehat{\mathbf{F}} \widehat{\mathbf{F}}^{T}$ with respect to the current basis is given by

$$
\widehat{\mathbf{B}}=\left[\begin{array}{ccc}
\frac{1}{\hat{\lambda}} & 0 & 0 \\
0 & \frac{1}{\hat{\lambda}}+r^{2} \hat{\gamma}^{2} & r \hat{\gamma} \hat{\lambda} \\
0 & r \hat{\gamma} \hat{\lambda} & \hat{\lambda}^{2}
\end{array}\right]
$$

Based on Eqs. 2.4 and 2.6 we may evaluate the Cauchy stress field, which must satisfy the equilibrium equation $\operatorname{div} \mathbf{T}=\mathbf{0}$. In particular, the only non trivial scalar equilibrium equation is

$$
\frac{d T_{r r}}{d r}+\frac{1}{r}\left(T_{r r}-T_{\theta \theta}\right)=0
$$

( $T_{i j}$ are the components of the Cauchy stress $\mathbf{T}$ in the current basis). By imposing the condition of unloaded lateral surface $\left(T_{r r}\left(r_{o}\right)=0\right)$ we get the expression

$$
T_{r r}(r)=-\int_{r}^{r_{o}}\left[\alpha \mu_{e} \rho \gamma^{2}+(1-\alpha) \mu_{b} \rho \int_{I(\rho)-\Delta}^{I(\rho)} \hat{\gamma}^{2} f\left(I_{a}\right) d I_{a}\right] d \rho,
$$

where $r_{o}:=R_{o} / \sqrt{\lambda}$ is the outer radius in the current configuration. By using Eq. 2.4 we obtain the pressure $p$ at $r$, i.e.,

$$
\begin{aligned}
p(r)= & \alpha \mu_{e}\left(\frac{1}{\lambda}+\frac{\gamma^{2}}{2}\left(r_{o}^{2}-r^{2}\right)\right) \\
& +(1-\alpha) \mu_{b}\left(\int_{I(r)-\Delta}^{I(r)} \frac{f\left(I_{a}\right)}{\hat{\lambda}} d I_{a}+\int_{r}^{r_{o}}\left(\rho \int_{I(\rho)-\Delta}^{I(\rho)} \hat{\gamma}^{2} f\left(I_{a}\right) d I_{a}\right) d \rho\right) .
\end{aligned}
$$


Thus, for a primary loading path described by Eq. 2.4, we can deduce the non-zero components of the Cauchy stress:

$$
\begin{aligned}
T_{r r}(r)= & \alpha \mu_{e} \frac{\gamma^{2}}{2}\left(r^{2}-r_{o}^{2}\right)-(1-\alpha) \mu_{b} \int_{r}^{r_{o}}\left(\rho \int_{I(r)-\Delta}^{I(r)} \hat{\gamma}^{2} f\left(I_{a}\right) d I_{a}\right) d \rho, \\
T_{\theta \theta}(r)= & \alpha \mu_{e} \frac{\gamma^{2}}{2}\left(3 r^{2}-r_{o}^{2}\right) \\
& +(1-\alpha) \mu_{b}\left[r^{2} \int_{I(r)-\Delta}^{I(r)} \hat{\gamma}^{2} f\left(I_{a}\right) d I_{a}-\int_{r}^{r_{o}}\left(\rho \int_{I(\rho)-\Delta}^{I(\rho)} \hat{\gamma}^{2} f\left(I_{a}\right) d I_{a}\right) d \rho\right], \\
T_{33}(r)= & \alpha \mu_{e}\left(\lambda^{2}-\frac{1}{\lambda}-\frac{\gamma^{2}}{2}\left(r_{o}^{2}-r^{2}\right)\right) \\
& +(1-\alpha) \mu_{b}\left[\int_{I(r)-\Delta}^{I(r)}\left(\hat{\lambda}^{2}-\frac{1}{\hat{\lambda}}\right) f\left(I_{a}\right) d I_{a}-\int_{r}^{r_{o}}\left(\rho \int_{I(\rho)-\Delta}^{I(\rho)} \hat{\gamma}^{2} f\left(I_{a}\right) d I_{a}\right) d \rho\right], \\
T_{3 \theta}(r)= & T_{\theta 3}(r)=\alpha \mu_{e} r \gamma \lambda+(1-\alpha) \mu_{b} r \int_{I(r)-\Delta}^{I(r)} \hat{\gamma} \hat{\lambda} f\left(I_{a}\right) d I_{a} .
\end{aligned}
$$

Finally, by using Eq. 3.3, we may evaluate the normal force $N:=2 \pi \int_{0}^{r_{o}} r T_{33}(r) d r$ and the twisting moment $M=2 \pi \int_{0}^{r_{o}} r^{2} T_{3 \theta}(r) d r$ acting on the bases of the cylinder:

$$
\begin{aligned}
& N=\alpha \mu_{e} \pi r_{o}^{2}\left(\lambda^{2}-\frac{1}{\lambda}-\frac{\gamma^{2} r_{o}^{2}}{4}\right) \\
&+2(1-\alpha) \mu_{b} \pi \int_{0}^{r_{o}} {\left[r \int_{I(r)-\Delta}^{I(r)}\left(\hat{\lambda}^{2}-\frac{1}{\hat{\lambda}}\right) f\left(I_{a}\right) d I_{a}\right.} \\
&\left.-\int_{r}^{r_{o}}\left(\rho \int_{I(\rho)-\Delta}^{I(\rho)} \hat{\gamma}^{2} f\left(I_{a}\right) d I_{a}\right) d \rho\right] d r \\
& M=\alpha \mu_{e} \frac{\pi}{2} \gamma \lambda r_{o}^{4}+2 \pi(1-\alpha) \mu_{b} \int_{0}^{r_{o}}\left(r^{3} \int_{I(r)-\Delta}^{I(r)} \hat{\gamma} \hat{\lambda} f\left(I_{a}\right) d I_{a}\right) d r .
\end{aligned}
$$

We remark that by using Eq. 2.6 the extensions of the expressions (3.3) and (3.4) to the case of unloading is simply obtained by replacing $I-\Delta$ by $I_{M}-\Delta$. Moreover, the response of an elastic Neo-Hookean material can be obtained by putting $\alpha=1$ in Eqs. 3.3 and 3.4. 


\section{Loading Cycles}

In the following we describe, through some numerical examples, the Mullins type softening behavior of the cylinder $\mathcal{B}$ for the class of deformations described in the previous section. To this end, we assume a given deformation history

$$
\lambda=\lambda(t), \quad \gamma=\gamma(t), \quad t \in(0, T),
$$

so that, in view of Eq. 3.1, we have

$$
I=I(R, t)=\frac{2}{\lambda(t)}+\lambda^{2}(t)+R^{2} \frac{\gamma^{2}(t)}{\lambda(t)} .
$$

First observe that we have to consider Eq. 3.3 for those points of the body with $I=I_{M}$; otherwise the expression Eq. 3.3 with $I-\Delta$ substituted by $I_{M}-\Delta$ must be used. In addition, the loading condition $\dot{I}>0$ depends on the point due to the $R$ dependence of $I$. However, if $(\lambda-1) \dot{\lambda}>0$ and $\gamma \dot{\gamma}>0$, then $\dot{I}>0$ for the whole cylinder. On the contrary, if $(\lambda-1) \dot{\lambda}<0$ and $\gamma \dot{\gamma}<0$, then $\dot{I}<0$ at each point.

In order to determine the axial force and the twisting moment, we consider the "instant" $t_{a}$ of activation of the breakable particles at $r$, defined by

$$
I\left(R, t_{a}\right)=I_{a}
$$

With this, Eq. 3.4 can be written in the form

$$
\begin{aligned}
N(t)= & \alpha \mu_{e} \pi r_{0}^{2}(t)\left(\lambda^{2}(t)-\frac{1}{\lambda(t)}-\frac{\gamma^{2}(t) r_{o}^{2}(t)}{4}\right) \\
& +2(1-\alpha) \mu_{b} \pi \int_{0}^{r_{o}(t)}\left[r \int_{\bar{t}(r)}^{t}\left(\frac{\lambda^{2}(t)}{\lambda^{2}\left(t_{a}\right)}-\frac{\lambda\left(t_{a}\right)}{\lambda(t)}\right) f\left(I\left(r, t_{a}\right)\right) \frac{d I\left(r, t_{a}\right)}{d t} d t_{a}\right. \\
& \left.-\int_{r}^{r_{o}}\left(\rho \int_{\bar{t}(\rho)}^{t}\left(\frac{\gamma(t)-\gamma\left(t_{a}\right)}{\lambda\left(t_{a}\right)}\right)^{2} f\left(I\left(\rho, t_{a}\right)\right) \frac{d I\left(\rho, t_{a}\right)}{d t} d t_{a}\right) d \rho\right] d r, \\
M(t)= & \alpha \mu_{e} \frac{\pi}{2} \gamma(t) \lambda(t) r_{o}^{4}(t) \\
& +2 \pi(1-\alpha) \mu_{b} \int_{0}^{r_{o}(t)}\left[r^{3} \int_{\bar{t}(r)}^{t}\left(\frac{\gamma(t)-\gamma\left(t_{a}\right)}{\lambda\left(t_{a}\right)} \frac{\lambda(t)}{\lambda\left(t_{a}\right)} f\left(I\left(r, t_{a}\right)\right) \frac{d I\left(r, t_{a}\right)}{d t}\right) d t_{a}\right] d r,
\end{aligned}
$$

where $\bar{t}(r)$ denotes the solution of the equation $I(\bar{t}(r), r)=I(t, r)-\Delta$ at the points with $I(r, t)=I_{M}(r)$, whereas it is the solution of $I(\bar{t}(r), r)=I_{M}(r)-\Delta$, otherwise. The solution $\bar{t}(r)$ denotes the "instant" at which the "most recently broken particle" has been activated, so that the particles that have been activated at $t<\bar{t}(r)$ are broken.

It is worth noting that the stress field depends on the whole loading history since the stress at each breakable particle depends on the values $\lambda\left(t_{a}\right)$ and $\gamma\left(t_{a}\right)$ attained at the activation time $t_{a}$. 


\subsection{Simple Extension}

First we consider the behavior of the cylinder $\mathcal{B}$ under a cyclic loading path of assigned stretch

$$
\lambda=\lambda(t)>1, \quad \gamma=\gamma(t)=0, \quad t \in(0, T) .
$$

For all details we refer to [4], where the possibility of determining the probability distribution $f$ by means of simple extension tests is also described. Here, we simply analyze the possibility of reproducing, through the proposed model, the Mullins behavior for simple extension loading cycles.

To this scope we observe that in the present case $I(t)=2 / \lambda(t)+\lambda^{2}(t)$. In particular, since for $t>0$ we assume $\lambda(t)>1, \dot{I}(t)>0$ when $\dot{\lambda}(t)>0$ and $\dot{I}(t)<0$ when $\dot{\lambda}(t)<0$. Moreover, $I_{M}=2 / \lambda_{M}+\lambda_{M}^{2}$ where $\lambda_{M}$ is the maximum past longitudinal stretch. Observe that since $\bar{t}$ does not depend on $r$, we get the following simplified expression of Eq. $4.2_{1}$ :

$$
\frac{N(t)}{\pi R_{o}^{2}}=\alpha \mu_{e}\left(\lambda(t)-\frac{1}{\lambda^{2}(t)}\right)+(1-\alpha) \mu_{b} \int_{\bar{t}}^{t}\left(\frac{\lambda(t)}{\lambda^{2}\left(t_{a}\right)}-\frac{\lambda\left(t_{a}\right)}{\lambda^{2}(t)}\right) f\left(I\left(t_{a}\right)\right) \frac{d I\left(t_{a}\right)}{d t} d t_{a} .
$$

In Figure $2 b$ we represent the normal force-stretch curve for a material defined by a Gaussian distribution function $f$ under cycles with increasing size (see Figure 2a). Curve 1 describes the primary loading path obtained during a loading starting from the virgin configuration with no previous breaking event. As the strain is increased an increasing percentage of material is activated at each point. In the figure we mark

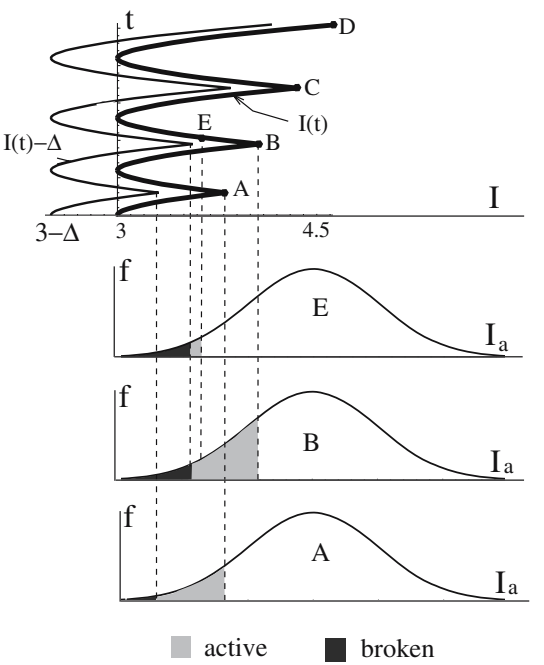

a)

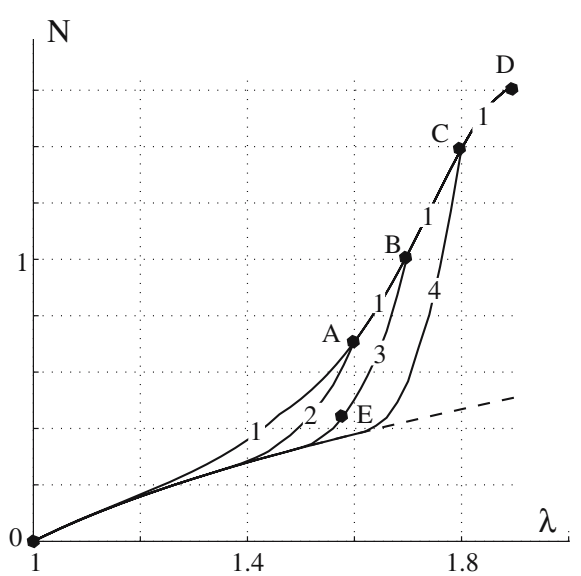

b)

Figure 2 Axial force-stretch cycles $(\gamma(t)=0)$. Here $\alpha=0.1, \mu_{e}=1, \mu_{b}=15$, and $f=e^{-\beta\left(I a-I_{o}\right)^{2}}$ with $\beta=15, I_{o}=4.5$ and $\alpha=0.8$. Finally, we assume $\Delta=0.5$ and $R_{o}=1$. In (a) we show the evolution of active and broken material fractions. In (b) we describe the corresponding force-stretch relationship. In (b) the dashed line represents the response of only the elastic percentage. 
with a grey area the percentage of activated material and with the black area the fraction of broken material in the probability space $f=f\left(I_{a}\right)$. The behavior is elastic until $I(t)=3+\Delta$, with the fraction of broken material $b=0$. When $I(t) \geq 3+\Delta$, a part of the activated material breaks.

We remark that in our model the (rubberlike) hardening effect is due to the increasing rate of growth of the activated fraction. Indeed, this effect is absent for the Neo-Hookean material as it is shown by the dashed line in Figure $2 \mathrm{~b}$ that represents the behavior of only the elastic fraction.

If the stress is decreased (e.g., path 2) the body exhibits the claimed softening effect: the normal force associated with each value of the stretch $\lambda$ decreases with the value of the previous maximum stretch $\lambda_{M}$. Observe that the body follows the same path (for $\lambda(t)<\lambda_{M}$ ) both for decreasing and increasing stretch (without hysteresis), describing the so called "ideal Mullins effect". We remark that during reloading the only active material is the percentage $\alpha$ of elastic material, until $I(t)=I_{M}-\Delta$ when a fraction of the breakable material is activated again. Thus, for $I(t)<I_{M}-\Delta$ all the curves coincide with the (dashed) curve describing the behavior of the sole elastic percentage.

\subsection{Pure Torsion}

In this section we consider the case of pure torsion: $\gamma=\gamma(t)$ and $\lambda(t)=1$. The Mullins effect for a circular cylinder subjected to pure torsion loading cycles has been also analyzed in [14]. In the present case, using Eq. 4.1, we have $I(t)=3+R^{2} \gamma^{2}(t)$ so that for $\gamma \dot{\gamma}>0$ it is $\dot{I}>0$. Moreover, since $I$ is a growing function of $R$ the damage grows with $R$, so that if $\gamma_{M}$ denotes the previous maximum value of the twist $\gamma$, by using Eq. 2.7 we have that

$$
R_{e}:=\frac{\sqrt{\Delta}}{\gamma_{M}}
$$

gives the radius of the elastic core of the cylinder (i.e., $b=0$ for $R \leq R_{e}$ ).

In the case of pure torsion, since $r=R$, Eq. 4.2 reduce to

$$
\begin{aligned}
N(t)= & -\alpha \mu_{e} \pi \frac{R_{o}^{4}}{4} \gamma^{2}(t) \\
& -2(1-\alpha) \mu_{b} \int_{0}^{R_{o}}\left[R \int_{R}^{R_{o}}\left(\rho \int_{\bar{t}(\rho)}^{t}\left(\gamma(t)-\gamma\left(t_{a}\right)\right)^{2} f\left(I\left(\rho, t_{a}\right)\right) \frac{d I\left(\rho, t_{a}\right)}{d t} d t_{a}\right) d \rho\right] d R, \\
M(t)= & \alpha \mu_{e} \frac{\pi}{2} \gamma(t) R_{o}^{4} \\
& +2 \pi(1-\alpha) \mu_{b} \int_{0}^{R_{o}}\left[R^{3} \int_{\bar{t}(R)}^{t}\left(\left(\gamma(t)-\gamma\left(t_{a}\right)\right) p\left(I\left(R, t_{a}\right)\right) \frac{d I\left(R, t_{a}\right)}{d t}\right) d t_{a}\right] d R .
\end{aligned}
$$

In Figure 3 we represent the numerical results under cyclic loads with increasing amplitude. Notice that, as a consequence of the Poynting effect, the deformation is maintained both by a moment $M$ and an axial force $N$. In particular, to have $\lambda$ constant, a compressive axial force is required. In this figure, we show for comparison 


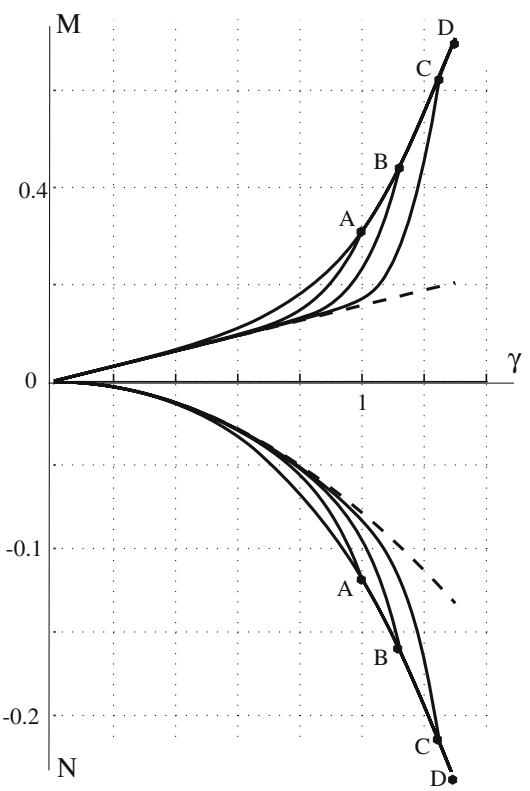

a)

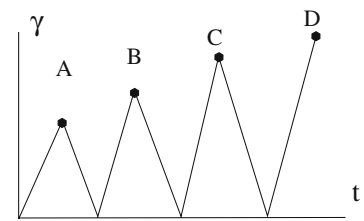

b)

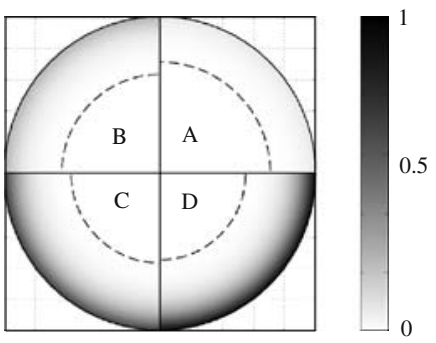

c)

Figure 3 Pure torsion cycles $(\lambda(t)=1)$ for the same material considered in Figure 2. In (a) we represent the twisting moment and the axial force as a function of $\gamma$ for the path represented in (b). In (a) the dashed lines represents the behavior of only the elastic fraction. In (c) we describe the evolution of the damage in a gray scale. In this figure the elastic core is bounded by a dashed line.

the response of only the elastic fraction $\alpha$ (dashed line). Observe that, as in the case of uniaxial extension, the rubberlike hardening is induced by the growing fraction of active material. Finally, in Figure $3 \mathrm{c}$ we represent the evolution of the damage $b$ at different points of the loading history. Here the elastic core is bounded by a dashed line. Of course, as the value $\gamma_{M}$ grows, the elastic core radius decreases.

\subsection{Combined Extension and Torsion}

In this section we consider the more general case of combined extension and torsion. The Mullins effect for the contemporary extension, torsion, and inflation of a tube has been analyzed in [8].

We first consider the behavior of the body under cyclic experiments with a proportional variation of $\lambda$ and $\gamma$

$$
\frac{\gamma(t)}{\lambda(t)-1}=\text { const. }
$$

The corresponding response curves $N-\lambda$ and $M-\gamma$, represented in Figure 4, show again the Mullins type softening behavior and the hardening effect associated with the increasing percentage of activated material. In the figure we also show the evolution of the damage $b$. Notice that, using Eqs. 4.1 and 2.7 we can evaluate the 'instant' 


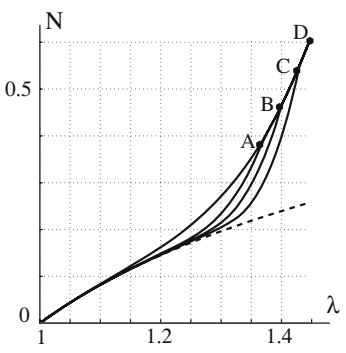

(a)

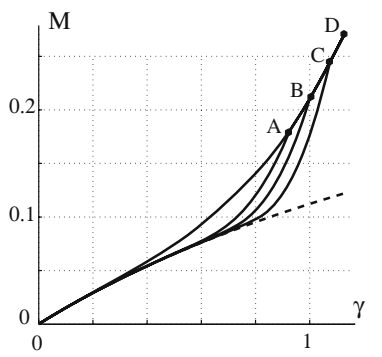

(b)

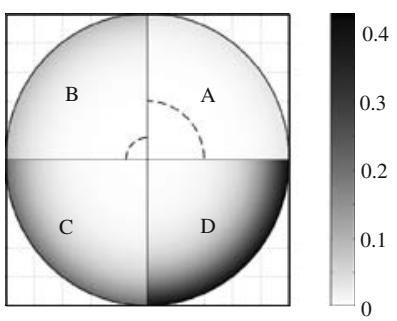

(c)

Figure 4 Proportional cycles with $\lambda(t)=1+t$ and $\gamma(t)=2.5 t$, for the same material considered in Figure 2. In (a) we represent the the axial force, in (b) the twisting moment, and in (c) the evolution of the damage $b$ in a gray scale. As in the previous figures, in (a) and (b) we also represent by dashed lines the response of the elastic fraction. In (c) the elastic core is bounded by a dashed line.

at which the elastic core disappears and the whole cylinder is damaged as the solution of $I(0, t)=3+\Delta$.

In Figure 5 we represent the remarkable path dependence of the cylinder. In particular, we consider three loading paths leading to the same deformed configuration $\left(\lambda_{M}, \gamma_{M}\right)$, so that we have the same final value of the damage $b$ at each point of the cylinder. Interestingly, we obtain different curves with a sensitive path dependence. We observe that the different values of $N$ and $M$ at $\gamma=\gamma_{M}$ and $\lambda=\lambda_{M}$ are a result of different stress fields. Indeed, as a consequence of the difference of the deformation histories, the particles are characterized by different activation deformation gradients $\mathbf{F}_{a}$ (see Section 2) in the three loading histories.

First, let us compare the $N-\lambda$ curves associated with the three paths (Figure $5 \mathrm{~b}$ ). In the path $O-A_{1}-A_{2}$, when first only the twist $\gamma$ increases $\left(\mathrm{O}-\mathrm{A}_{1}\right)$ we observe a compressive normal force due to the Poynting effect; later when the stretch is increased $\left(A_{1}-A_{2}\right)$, this path is characterized by the largest stiff ness due to the largest value of activated material fraction. The other two paths have a less pronounced difference produced by the simultaneous presence of Poynting effect and variable damage and activation.

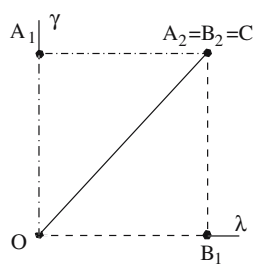

(a)

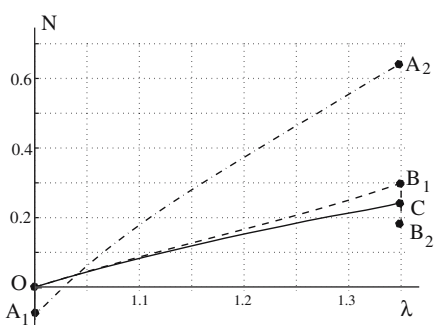

(b)

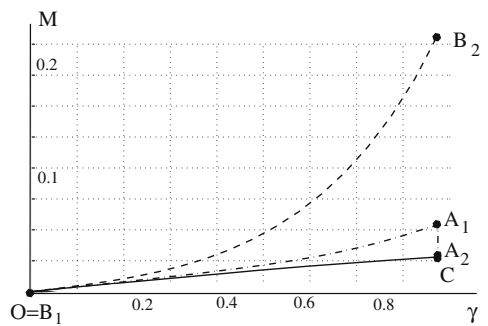

(c)

Figure 5 Path dependent behavior for the same material studied in Figure 2. In (a) we represent the three different loading paths and in (b) and (c) the corresponding different force-elongation and moment-twist diagrams. 
Figure 6 Preconditioning effects for the same material of Figure 2. In the figure we represent the effect in the $M-\gamma$ and $N-\gamma$ diagrams due to previous cycles $\lambda=1 \rightarrow \lambda_{M} \rightarrow 1$ for different values of $\lambda_{M}$ (curve 0 is the virgin curve, curve 1 corresponds to $\lambda_{M}=1.5$, curve 2 to $\lambda_{M}=1.6$, and curve 3 to $\left.\lambda_{M}=1.7\right)$.

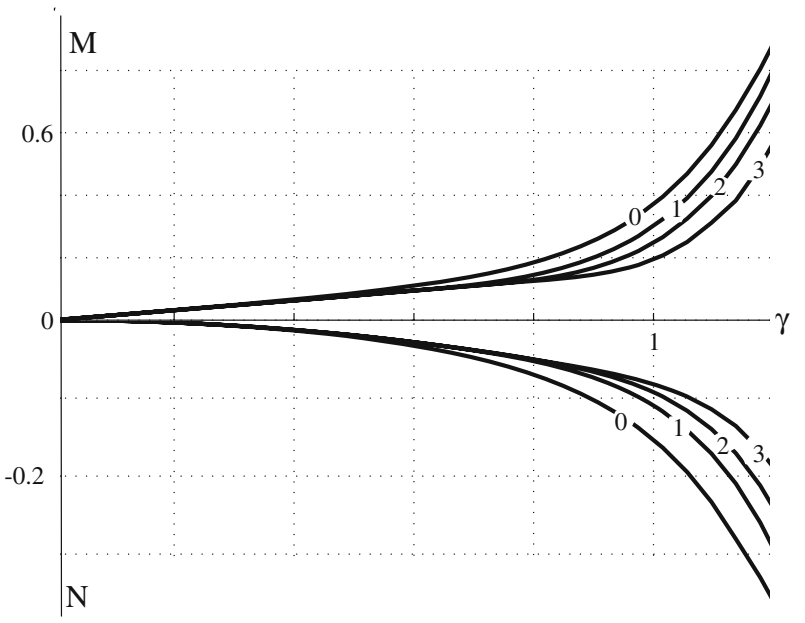

Similar considerations can be extended to the twisting moment curves in Figure $5 \mathrm{c}$. Here the stiffest behavior is given by the path $\mathrm{O}-\mathrm{B}_{1}-\mathrm{B}_{2}$ characterized by a previous pure extension.

Finally, in Figures 6 and 7 we show the preconditioning effects exhibited by our model. In particular, in Figure 6 we consider the effect of previous loading cycles of simple extension with growing sizes on the pure torsion deformation. Whereas the case of pure extension preconditioned by previous torsion cycles with different amplitude is represented in Figure 7. Notice that in Figure 6 the preconditioned curves don't reconnect at "large" $\gamma$ to the virgin curve. This behavior must be addressed to the observation that while the virgin curve is characterized by the presence of an elastic core with no damage, the other curves start from a homogeneous damage configuration, due to the preconditioning of the normal force, with no elastic core. On the contrary, in the case of preconditioning by means of torsion cycles (see Figure 7), we have that for 'large' values of $\lambda$ the damage of the preconditioned and virgin curves coincide so that the curves reconnect.

Figure 7 Preconditioning effects for the same material of Figure 2. In the figure we show the softening effect in the $N-\lambda$ curves due to previous cycles of torsion $\gamma=1 \rightarrow \gamma_{M} \rightarrow 1$ for different values of $\gamma_{M}$. Curve 0 is the virgin curve, curve 1 corresponds to $\gamma_{M}=0.5$, curve 2 to $\gamma_{M}=0.6$, and curve 3 to $\gamma_{M}=0.7$.

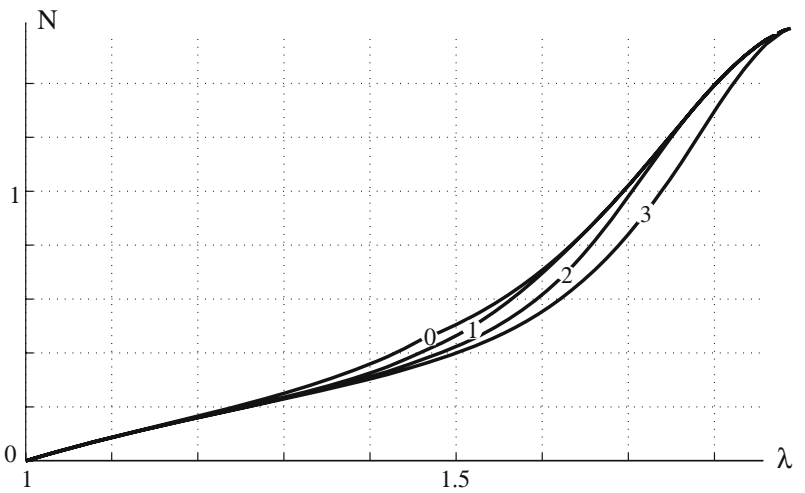


Acknowledgements This work was supported by the research projects MIUR-PRIN 2005 "Strutture in materiali con microstruttura. Una sfida per la moderna ingegneria civile" and "Progetto Strategico, Regione Puglia: Metodologie innovative per la modellazione e la sperimentazione sui materiali e sulle strutture, finalizzate all'avanzamento dei sistemi produttivi nel settore dell'Ingegneria Civile."

\section{References}

1. Beatty, M.F., Krishnaswamy, S.: The Mullins effect in equibiaxial deformation. J. Appl. Math. Phys. (ZAMP) 51, 984-1015 (2000)

2. Beatty, M.F., Krishnaswamy, S.: A theory of stress-softening in incompressible isotropic materials. J. Mech. Phys. Solids 48, 1931-1965 (2000)

3. Bouasse, H., Carrière, Z.: Courbes de traction du caoutchouc vulcanisé. Ann. Fac. Sci. Toulouse 5, 257-283 (1903)

4. De Tommasi, D., Puglisi, G., Saccomandi, G.: A micromechanics based model for the Mullins effect. J. Rheol. 50, 495-512 (2006)

5. Horgan, C.O., Ogden, R.W., Saccomandi, G.: A theory of stress softening of elastomers based on finite chain extensibility. Proc. R. Soc. Lond. A 460, 1737-1754 (2004)

6. Horgan, C.O., Saccomandi, G.: Pure azimuthal shear of isotropic, incompressible hyperelastic materials with limiting chain extensibility. Int. J. Nonlin. Mech. 36, 465-475 (2001)

7. Krishnaswamy, S., Beatty, M.F.: The Mullins effect in compressible solids. Int. J. Eng. Sci. 38, 1397-1414 (2000)

8. Krishnaswamy, S., Beatty, M.F.: Damage induced stress-softening in the torsion, extension and inflation of a cylindrical tube. J. Mech. Appl. Math. 54, 295-327 (2001)

9. Mullins, L.: Effect of stretching on the properties of rubber. J. Rubber Res. 16, 275-289 (1947)

10. Mullins, L.: Softening of rubber by deformation. Rubber Chem. Technol. 42, 339-362 (1969)

11. Mullins, L., Tobin, N.: Theoretical model for the elastic behavior of filler-reinforced vulcanized rubbers. Rubber Chem. Tech. 30, 551-571 (1953)

12. Rajagopal, K.R., Srinivasa, A.R.: On the thermomechanics of materials that have multiple natural configurations. Part I. J. Appl. Math. Phys. (ZAMP) 55, 861-893 (2004)

13. Rajagopal, K.R., Wineman, S.: A constitutive equation for nonlinear solids which undergo deformation induced microstructural changes. Int. J. Plast. 8, 385-395 (1992)

14. Wineman, S.: Torsion of an elastomeric cylinder undergoing microstructural changes. J. Elast. 62, 217-237 (2001)

15. Wineman, S., Huntley, H.E., Rajagopal, K.R.: Chemorheological relaxation, residual stress and permanent set arising in radial deformations of elastomeric hollow spheres. Math. Mech. Solids 1, 267-300 (1996)

16. Wineman, S., Min, J.: Time dependent scission and cross-linking in an elastomeric cylinder undergoing circular shear and heat conduction. Int. J. Nonlinear Mech. 38, 969-983 (2003)

17. Wineman, S., Rajagopal, K.R.: On a constitutive theory for materials undergoing microstructural changes. Arch. Mech. 42, 53-57 (1990) 